\title{
The ATLAS Detector: Performance Results
}

\author{
I. Riu* \\ IFAE, Universitat Autònoma de Barcelona, Bellaterra, Spain \\ E-mail: Imma.Riu@cern.ch
}

\section{On behalf of the ATLAS Collaboration}

The ATLAS experiment is designed to study the proton-proton collisions produced at the Large Hadron Collider at CERN. During the Run 1 data-taking period, the ATLAS trigger and data acquisition system has been used to collect a large data sample useful for a wide variety of physics analyses. The 2012 run saw both larger luminosity and larger number of simultaneous interactions than in 2011, challenging the trigger system, object reconstruction and physics analyses. The efficient reconstruction and identification of the different leptons, $b$-quark jets and missing transverse energy played a key role in the different analyses, leading to the discovery of the Higgs boson in July 2012.

This note reports about efficiency, resolution, and general performance of the ATLAS detector by using mainly proton-proton collisions at $\sqrt{s}=8 \mathrm{TeV}$ collected in 2012 .

The European Physical Society Conference on High Energy Physics -EPS-HEP2013

18-24 July 2013

Stockholm, Sweden

${ }^{*}$ Speaker. 


\section{Introduction}

In February 2013, the ATLAS detector [1] recorded the last data from the Run 1 period before entering into the Long Shutdown 1 activities. During its three years of operation, ATLAS succeeded recording proton-proton collisions at centre-of-mass energies ranging from $0.9 \mathrm{TeV}$ up to $8 \mathrm{TeV}$ in addition to recording lead-lead and proton-lead ions collisions. In 2012, a record peak instantaneous luminosity of $7.73 \times 10^{33} \mathrm{~cm}^{-2} \mathrm{~s}^{-1}$ was achieved largely contributing to record a total integrated luminosity of $23.3 \mathrm{fb}^{-1}$ at the end of the year. Together with the luminosity increase in 2012, the pile-up, the number of simultaneous inelastic proton-proton interactions per bunch crossing, also increased to between 10 and 20 interactions on average. Pile-up doesn't only affect detector operations and trigger but also data reconstruction, object identification and isolation.

The trigger and data acquisition overall performance in 2012 was very good with an uptime for all sub-detectors of more than $99 \%$. The average data acquisition efficiency was $\sim 93.5 \%$. ATLAS succeeded to run with a very small number of non-operational detector channels. The trigger system recorded data separately according to trigger-reconstructed objects like: electrons and photons; jets, taus and missing transverse energy; and muons, among others. On average, $400 \mathrm{~Hz}$ of proton-proton collisions were recorded in 2012. After data quality requirements, about $95.5 \%$ of the recorded data was good for physics analyses. This note focuses mainly on the general performance of the ATLAS detector under the pile-up conditions experienced during the 2012 data taking period.

\section{The ATLAS detector}

The ATLAS detector at the Large Hadron Collider covers almost the entire solid angle around the nominal interaction point. It consists of an inner tracking detector surrounded by a superconducting solenoid magnet producing a $2 \mathrm{~T}$ axial magnetic field; electromagnetic and hadronic calorimeters providing accurate energy and momentum measurements; and an external muon spectrometer incorporating three large toroid magnet assemblies. The inner detector consists of a high-granularity silicon pixel detector and a silicon microstrip tracker, together providing precision tracking in the range $|\eta|<2.5^{1}$, complemented by a transition radiation tracker providing tracking and electron identification information for $|\eta|<2.0$. A lead liquid-argon (LAr) electromagnetic calorimeter covers the region $|\eta|<3.2$, and hadronic calorimetry is provided by steel/scintillating tile calorimeters for $|\eta|<1.7$ and copper/LAr hadronic endcap calorimeters. The forward region is covered by additional LAr calorimeters with copper and tungsten absorbers. The muon spectrometer consists of precision tracking chambers covering the region $|\eta|<2.7$, and separate trigger chambers covering $|\eta|<2$.4. A three-level trigger system, using custom hardware followed by two software-based levels, is used to select interesting events for recording and subsequent offline analysis.

\section{Tracking and vertex performance}

Several pattern-recognition strategies are used to reconstruct tracks in the inner detector. The

\footnotetext{
${ }^{1}$ ATLAS uses a right-handed coordinate system with its origin at the nominal interaction point in the centre of the detector, and the $z$-axis along the beam line. Pseudorapidity is defined in terms of the polar angle $\theta$ as $\eta=-\ln \tan (\theta / 2)$.
} 

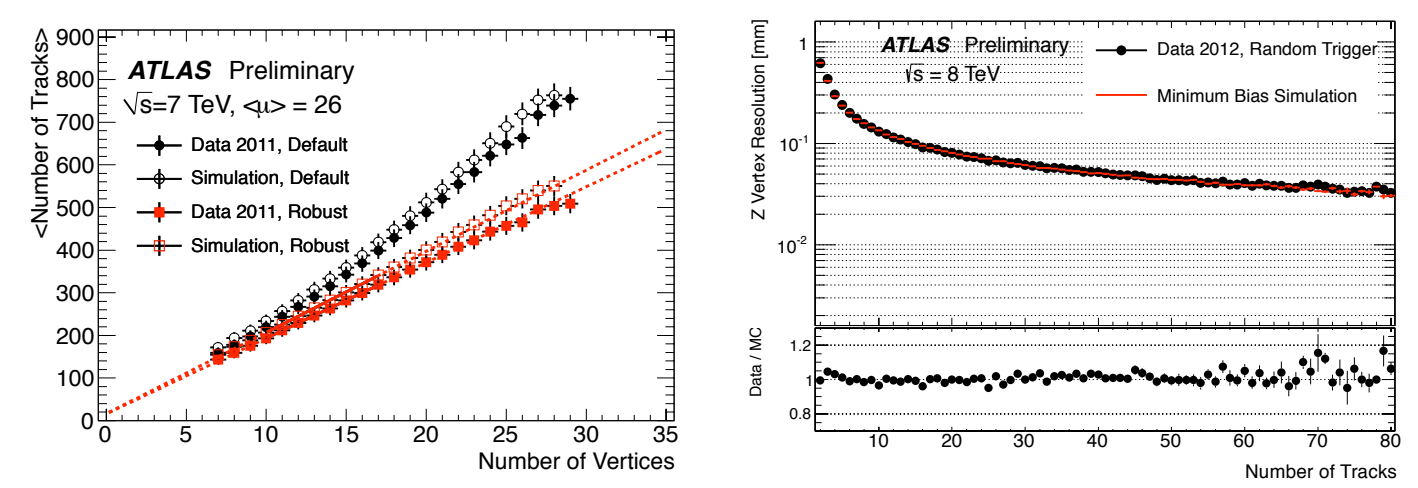

Figure 1: On the left, the average number of tracks per event as a function of the number of vertices [2]. On the right, the resolution of the vertex longitudinal coordinate as a function of the number of tracks used in the vertex fit.

"inside-out" approach reconstructs charged tracks with $p_{\mathrm{T}}>400 \mathrm{MeV}$. It starts in the two silicon systems and tracks are extended to higher radii. The reverse approach, referred to as "backtracking", is used to reconstruct tracks originating mainly from photon conversions or long-lived particles decays. In the presence of multiple interactions and high detector channel occupancy in 2012, the number of fake tracks reconstructed from random combinations of inner detector measurements increased dramatically. To reduce them and increase stability, a high number of hits in the silicon and no holes in the pixel were required for tracks, designated as the Robust method [2], while less stringent criteria were used in 2011, designated as Default. The left plot of Fig. 1 shows the average number of tracks per event as a function of the number of vertices: the number of tracks reduces with the Robust criteria compared to the Default one. Iterative vertex finding and fitting algorithms [2] are used with tracks fulfilling basic track quality. The longitudinal vertex resolution is shown on the right of Fig 1 and is better than $\sim 40 \mu \mathrm{m}$ when at least 50 tracks are used in the vertex fit. Data agree well with Monte Carlo simulation.

\section{Electron and photon performance}

Thanks to frequent calibrations of the LAr calorimeter, excellent stability of the energy scale both over time and with pile-up was achieved. Electron candidates are identified using shower shape characteristics combined with tracking information. Three reference sets of cuts are defined with increasing background rejection power: loose, medium and tight. Using a tag-and-probe method to a selected sample of $Z$ boson decays into a pair of electrons, efficiencies for each category are measured to be 95,88 and $70 \%$ respectively [3]. The electron identification efficiency pileup dependence for each electron reference is compared to simulation on the left of Fig. 2. No dependence is observed up to 20 primary vertices.

Making use of the electromagnetic calorimeter longitudinal fine segmentation prompt photons are discriminated from photons originating for example from jets with a leading $\pi^{0}$ decay. Methods to identity both converted and unconverted photons were developed in ATLAS [4]. Using a sample of radiative decays of the $Z$ boson including both $Z \rightarrow e e \gamma$ and $Z \rightarrow \mu \mu \gamma$, the photon efficiency is measured. The efficiency of unconverted photons up to $|\eta|<0.6$ is shown on the right plot of 

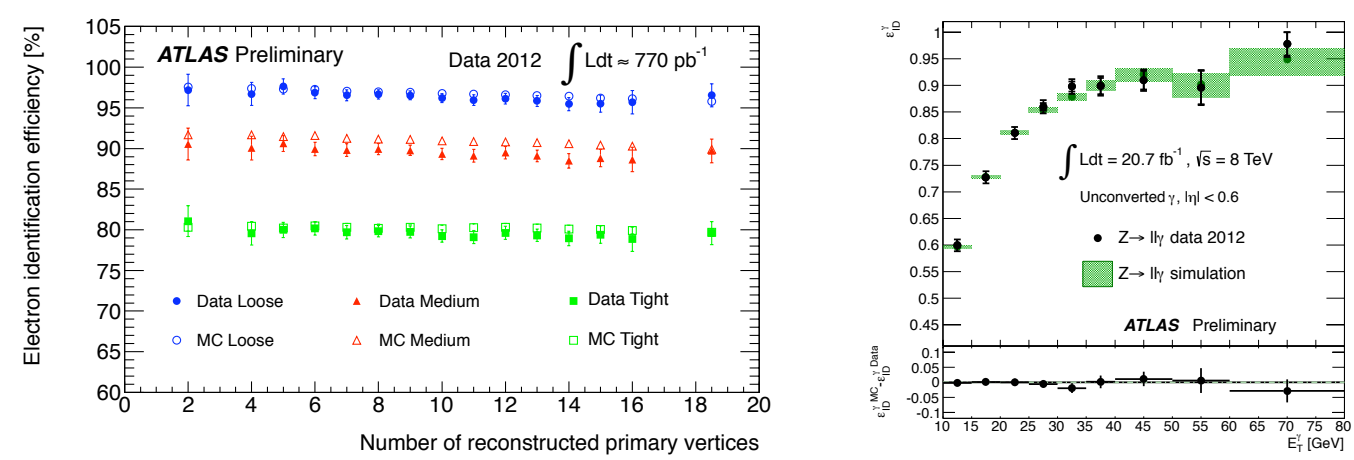

Figure 2: On the left, electron efficiency measurements as a function of the number of reconstructed primary vertices for different electron categories. On the right, efficiency of unconverted photons up to $|\eta|<0.6$ as a function of the transverse photon energy, with its difference to Monte Carlo shown at the bottom.

Fig. 2 as a function of the photon transverse energy. Data and Monte Carlo simulation are in good agreement.

\section{Jet and missing transverse energy performance}

Periodic monitoring of the tile calorimeter cells was performed during data taking and cell energies of better than $1 \%$ on the electromagnetic energy scale were measured. Both jets and transverse missing energy performance suffered from the large pile-up conditions in 2012. A new pile-up subtraction method to correct the four momentum of the jet event-by-event was developed in 2012. It is based on the jet area, $A$, (measured jet-by-jet) [5] and the median $p_{\mathrm{T}}$ density $\rho$, a measure of the pile-up activity in the event. As shown on the left of Fig. 3, this method reduces the RMS width of the jet $p_{\mathrm{T}}$ difference between reconstruction and truth when compared to the method that applies an average correction parametrized by the number of primary vertices $\mathrm{N}_{\mathrm{PV}}$ and average pile-up $\langle\mu\rangle$.

Concerning the missing transverse energy, a pile-up suppression method based on the ratio of the sum $p_{\mathrm{T}}$ of the tracks associated to the primary vertex and all tracks was applied. Its effect can be observed on the right of Fig. 3 for $Z \rightarrow \mu \mu$ Monte Carlo events and data candidates. After pile-up suppression, the resolution of the $x$ and $y$ missing transverse energy components show very little dependence on pile-up.

\section{6. $b$-tagging and tau performance}

The identification of jets originated from $b$-quarks ( $b$-jets), commonly known as $b$-tagging, is very important for the ATLAS physics program. Different algorithms making use of multi-variate discriminants were developed to identify $b$-jets. Their performance is assessed by measuring the rejection of light-flavour jets as a function of the $b$-jet efficiency. The most commonly used $b$ tagging algorithm in ATLAS is the multi-variate algorithm MV1 [8].

Hadronically decaying tau leptons also play an important role in physics analyses in ATLAS. Their identification is difficult because their detector signature is very similar to the signature as- 

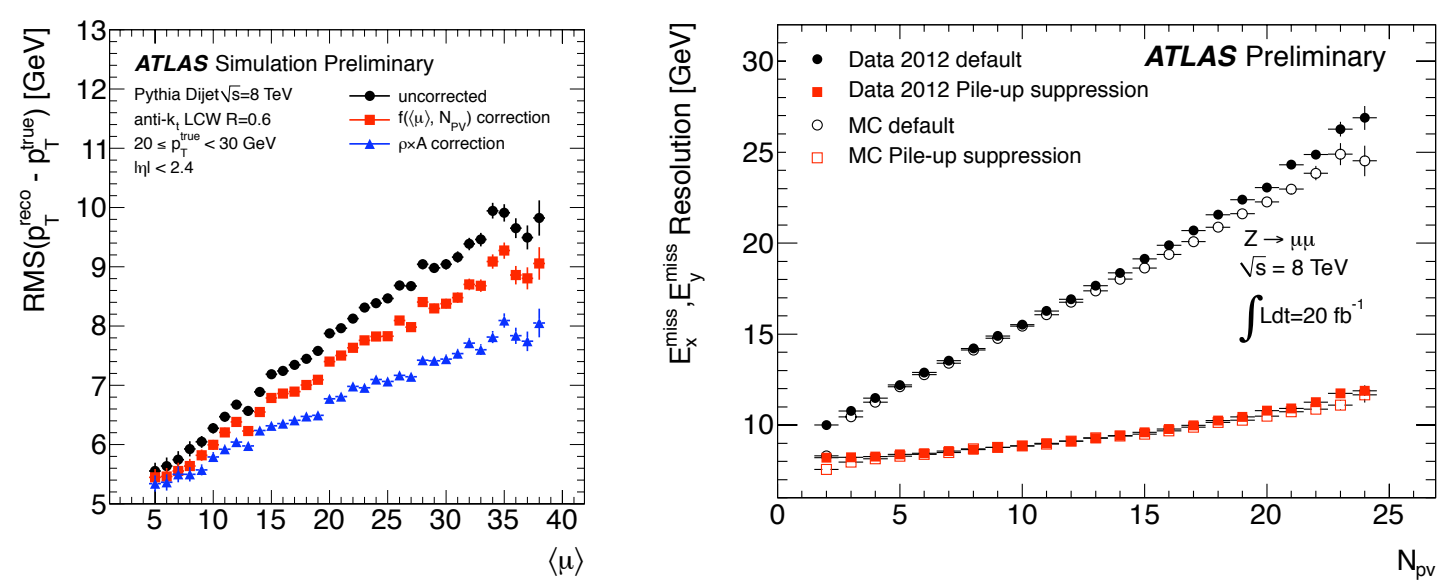

Figure 3: On the left, RMS width of the jet $p_{\mathrm{T}}^{\text {reco }}-p_{\mathrm{T}}^{\text {true }}$ distribution as a function of the average pile-up $\langle\mu\rangle$ in simulated dijet events [6]. On the right, resolution of $x$ and $y$ missing transverse energy components as a function of the number of primary vertices for $Z \rightarrow \mu \mu$ data candidates and Monte Carlo events [7].
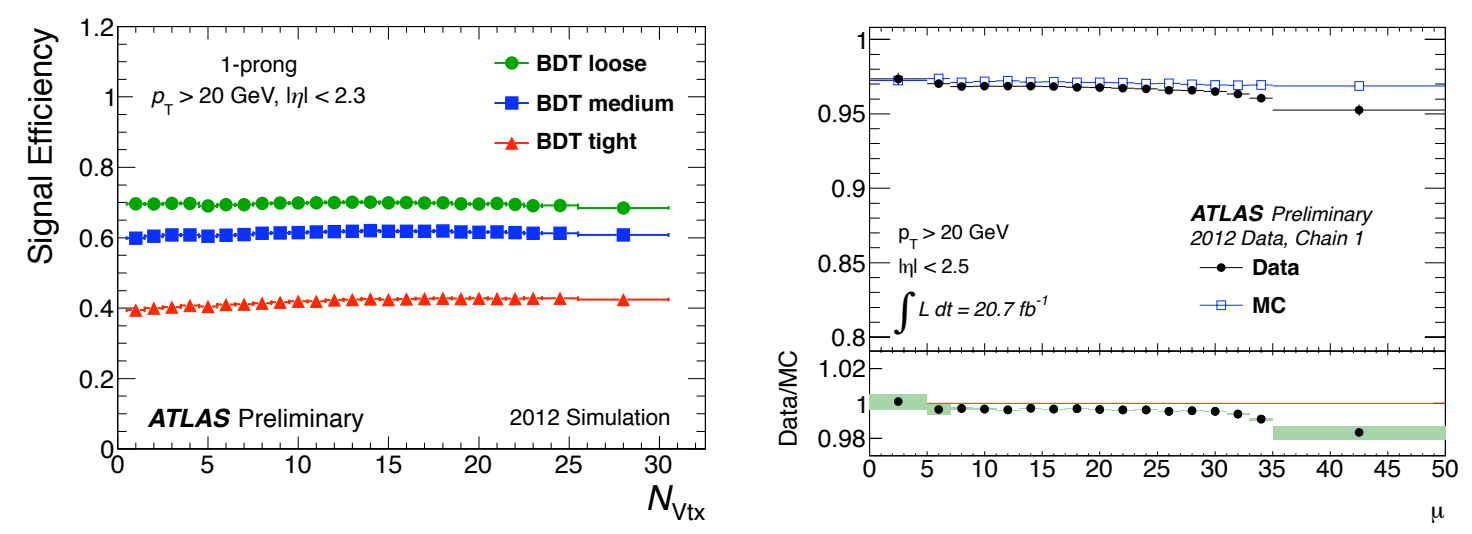

Figure 4: On the left, signal efficiency for one-prong hadronic tau candidates for the three working points of the BDT tau identification as a function of the number of vertices [10]. On the right, muon reconstruction efficiency for muons with $p_{\mathrm{T}}>20 \mathrm{GeV}$ versus the number of pile-up interactions. The panel at the bottom shows the ratio between the measured and predicted efficiencies.

sociated with jets or electrons. Characteristic to hadronic tau lepton decays are narrow calorimeter deposits as well as few tightly spaced tracks. Dedicated multi-variate techniques such as a projective likelihood method or boosted decision trees (BDT) [9] are used to discriminate hadronic taus from jets and other leptons. Different tau identification working points corresponding to decreasing target efficiencies are defined. The signal efficiency for one-prong tau candidates (with one reconstructed track) for the three working points of the tau identification with BDT as a function of the number of vertices is shown on the left of Fig. 4. It is obtained using simulated samples of $Z$ and $W$ boson decays to hadronic taus. It shows almost no dependence on pile-up. 


\section{Muon performance}

Different reconstruction methods using standalone or various combinations of sub-detectors were used to reconstruct muon candidates in the 2012 data sample. Through selected samples of both $Z$ boson and $J / \psi$ meson decays, a tag-and-probe method is applied to measure the muon reconstruction efficiency. Data and Monte Carlo muon reconstruction efficiency differences in the range of only 0.1 to $1 \%$ are found. The muon momentum were found to be reasonably well simulated and scale corrections of only $\sim 0.1 \%$ were applied, leading to a very good data versus Monte Carlo agreement of the di-muon invariant mass distribution. As shown on the right of Fig. 4, the muon reconstruction efficiency has no dependence on event pile-up.

\section{Conclusions}

During the period of Run 1, ATLAS acquired a large sample of proton-proton collisions with high efficiency, about $95 \%$ of which are good for data analyses. Large number of studies were performed in order to improve the performance of physics objects reconstruction and identification against the large pile-up conditions present in 2012. Overall good performance as well as results compatible with Monte Carlo predictions were achieved. This work largely contributed to the discovery of the Higgs boson announced in July 2012.

\section{References}

[1] ATLAS Collaboration, JINST 3 (2008) S08003.

[2] ATLAS Collaboration, ATLAS-CONF-2012-042, http://cdsweb.cern.ch/record/1435196.

[3] ATLAS Collaboration, Eur. Phys. J. C 721909 (2012).

[4] ATLAS Collaboration, Phys. Rev. D 83, 052005 (2011).

[5] M. Cacciari, G. P. Salam, and G. Soyez, JHEP 0804 (2008) 005, arXiv:0802.1188 [hep-ph].

[6] ATLAS Collaboration, ATLAS-CONF-2013-083, http://cdsweb.cern.ch/record/1570994.

[7] ATLAS Collaboration, http://twiki.cern.ch/twiki/bin/view/AtlasPublic/JetEtmissApproved2013EtMiss.

[8] ATLAS Collaboration, ATLAS-CONF-2012-043, http://cdsweb.cern.ch/record/1435197.

[9] ATLAS Collaboration, ATLAS-CONF-2011-077, http://cdsweb.cern.ch/record/1353226.

[10] ATLAS Collaboration, ATLAS-CONF-2013-064, http://cdsweb.cern.ch/record/1562839. 